\title{
PD-L1/PD-1 blockade in breast cancer: The immunotherapy era (Review)
}

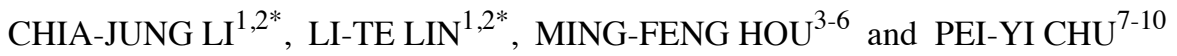 \\ ${ }^{1}$ Department of Obstetrics and Gynecology, Kaohsiung Veterans General Hospital, Kaohsiung 813; \\ ${ }^{2}$ Institute of BioPharmaceutical Sciences, National Sun Yat-sen University, Kaohsiung 804; ${ }^{3}$ Division of Breast Surgery, \\ Department of Surgery, Center for Cancer Research, Kaohsiung Medical University Chung-Ho Memorial Hospital; \\ ${ }^{4}$ Graduate Institute of Clinical Medicine, Kaohsiung Medical University; ${ }^{5}$ National Sun Yat-Sen University-Kaohsiung \\ Medical University Joint Research Center, Kaohsiung Medical University; ${ }^{6}$ National Chiao Tung University-Kaohsiung \\ Medical University Joint Research Center, Kaohsiung Medical University, Kaohsiung 807; ${ }^{7}$ School of Medicine, \\ College of Medicine, Fu Jen Catholic University, New Taipei 242; ${ }^{8}$ Department of Pathology, Show Chwan Memorial Hospital, \\ Changhua 500; ${ }^{9}$ Department of Health Food, Chung Chou University of Science and Technology, Changhua 510; \\ ${ }^{10}$ National Institute of Cancer Research, National Health Research Institutes, Tainan 704, Taiwan, R.O.C.
}

Received July 9, 2020; Accepted August 30, 2020

DOI: 10.3892/or.2020.7831

\begin{abstract}
Breast cancer is a common malignant tumor in women. Triple-negative breast cancer (TNBC) is highly invasive with a high rate of metastasis and poor prognosis. Programmed death ligand 1 (PD-L1) plays an important role in mediating the escape of tumor cells from immune surveillance. There have been significant advances in understanding the biology of TNBC. This review presents a detailed discourse on the available data on the expression of PD-L1 in breast cancer and preliminary clinical outcome of PD-L1/PD-1 inhibitors in breast cancer patients. Early clinical trials involving PD-L1/PD-1 inhibitors have exhibited efficacy in tumor response and/or disease control in patients with refractory metastatic breast cancer, particularly TNBC. Furthermore, the mechanisms and factors that influence the immunoediting process are summarized and their functions in detail are analyzed.
\end{abstract}

\section{Contents}

1. Introduction

2. Overview of tumor immunotherapy

3. Biological characteristics of PD-1/PD-L1

Correspondence to: Professor Pei-Yi Chu, Department of Pathology, Show Chwan Memorial Hospital, 542, Sec. 1, Chung-Shang Road, Changhua 500, Taiwan, R.O.C.

E-mail: chu.peiyi@msa.hinet.net

*Contributed equally

Key words: breast cancer, programmed cell death-1, programmed death ligand 1 , immunotherapy
4. Prognostic significance of PD-1/PD-L1

5. Significance of targeting PD-1/PD-L1 monoclonal antibody

6. Anti-PD-L1 therapy for breast cancer

7. Future perspectives

\section{Introduction}

Breast cancer has been identified as one of the leading causes of cancer-related deaths worldwide. Abnormalities in proteins result in malignant transformation of cells that results in 25-40\% of recurrence and metastasis of cancer (1). Breast cancer is a common malignant tumor and its rate of incidence was the highest among tumors detected in women in 2019 (2).

Approximately 627,000 people are expected to succumb to breast cancer in 2019. Due to the lack of specific therapeutic targets available for triple-negative breast cancer (TNBC), the prognosis of TNBC remains unsatisfactory. The emergence of anti-PD-L1/PD-1 therapeutics has shown promise for the treatment of TNBC (3). In the present study, the advancements in the efficacy of PD-L1 against TNBC are described. Programmed death ligand 1 (PD-L1) is a ligand of programmed cell death-1 (PD-1) and is expressed on various tumors and immune cells. Once PD-L1 binds to PD-1, it inhibits T-cell migration and proliferation and the secretion of cytotoxic mediators, thereby limiting its killing effect on tumor cells (3). PD-L1 has been revealed to be highly expressed in a variety of tumors and enhance antitumor immunity by inhibiting PD-L1 (4). The development and clinical application of targeted drugs against signaling pathways associated with the occurrence and development of breast cancer have become a hot spot in research on breast cancer treatment (5). Targeted therapy has better clinical efficacy and safety than cytotoxic drugs (6). The present study reviewed the current molecular targeted therapeutics in treating breast cancer. 


\section{Overview of tumor immunotherapy}

Tumor immunotherapy is a method of treatment that has been developed in recent years. It stimulates and regulates immune function, enhances antitumor ability, and controls and kills tumor cells (7). Immunotherapy has attracted attention owing to the importance of antitumor immunity, diverse mechanisms in escape of tumors from immune surveillance, continuous discovery of novel therapeutic targets and immunotherapies $(8,9)$. Tumor immunotherapy can be divided into active and passive immunotherapy. Active immunotherapy employs tumor vaccines to mimic tumor antigens to activate immune effects on tumors, thereby directly or indirectly promoting specific antitumor immune responses in humans (10). Passive immunotherapy includes the use of monoclonal antibodies, adoptive immune cells, and cytokines. Monoclonal antibody therapy involves the administration of specific antibodies to stimulate host immune response against tumor antigens $(11,12)$. Adoptive immune cell therapy separates and expands immune cells to induce the production of cytokines before introducing the cells into patients to increase the abundance of immune cells and enhance their anticancer function (13). Cytokine therapy involves the direct injection of cytokines into the human body, which enhances antitumor function in immune cells $(14,15)$. Compared to traditional methods of treatment, tumor immunotherapy has specific advantages: Tumor immune-related monoclonal antibodies and tumor vaccines exhibit fewer adverse reactions, strong specificity, and promising clinical application (13).

An increase in research on immune checkpoints has been observed in recent years. Immune checkpoints are inhibitory signaling pathways present in the immune system that regulate the persistence and intensity of immune response, maintain autoimmune tolerance, and avoid tissue damage $(16,17)$. Dysfunction of key negative regulatory molecules during T-cell activation is important for the tolerance and escape of tumor immunity. Inhibition of the immune checkpoint can reverse the immunosuppressive state of the tumor microenvironment and enhance the clearance of tumor cells (18). Immunological checkpoints include cytotoxic $\mathrm{T}$ lymphocyte-associated antigen-4 (CTLA-4), PD-1, B and T lymphocyte attenuator (BTLA), and lymphocyte activation gene 3 (LAG3). The FDA-approved immunological checkpoint inhibitor, ipilimumab, that targets CTLA-4 is used to treat melanoma $(19,20)$. Researchers are further identifying novel therapeutic targets to regulate immune checkpoints. The most important monoclonal antibody currently in use targets the immune checkpoint PD-1 and its ligand PD-L1 (21). PD-L1/PD-1-related immunotherapy has become a hotspot for research on tumor immunotherapy.

\section{Biological characteristics of PD-1/PD-L1}

PD-1 is a type I transmembrane protein expressed on the surface of activated $\mathrm{T}$ cells, B cells, monocytes and dendritic cells and is composed of extracellular, hydrophobic transmembrane, and cytoplasmic regions (22). Its extracellular domain consists of a single IgV-like domain with an immunoreceptor tyrosine-based inhibitory motif (ITIM) and immunoreceptor tyrosine-based motif (23). ITIM can be commonly found in numerous immunosuppressive receptors (24). The immunosuppressive function of PD-1 is primarily exerted via ITIM. PD-L1 and PD-L2 are the ligands of PD-1 (25). PD-L1 is the primary ligand that is upregulated in various solid tumors. This reduces the infiltration of $\mathrm{CD}^{+}$and $\mathrm{CD}^{+} \mathrm{T}$ cells into tumors and concomitant cytokine production. PD-L1 is a member of the B7 superfamily and a type I transmembrane glycoprotein $(26,27)$. PD-L1 is expressed in various tumors, such as urothelial, ovarian, breast, cervical, colorectal, pancreatic, gastric, melanoma, malignant glioma, and non-small cell lung cancers among others. This suggests the involvement of PD-1 signaling in tumors for immune evasion (7,28-30). PD-1/PD-Ls exerts a negative immunomodulatory effect. The surface of tumor cells in the tumor microenvironment exhibit increased expression of PD-L1 that binds to PD-1 on activated T cells, which leads to apoptosis or immunological inactivation of tumor antigen-specific $\mathrm{T}$ cells, thereby inhibiting immune response and promoting the evasion of tumor cells (31).

PD-L1 is expressed on the surface of various tumor and immune cells, such as T, B, and dendritic cells (13). Tumor cells bind to PD-1 on the surface of tumor infiltrating lymphocytes (TILs) via PD-L1. Activation of TILs enables immunosuppressive signaling, inhibits T-cell migration and proliferation, secretion of cytotoxic mediators, induces T-cell depletion, and limits its antitumor effects, thereby resulting in immune evasion $(32,33)$. Decreased binding of PD-L1 to PD-1 reverses immune escape, enhances antitumor immunity, and inhibits tumor progression (34). However, PD-L1/PD-1-targeted immunotherapy has been revealed to be effective in clinical trials of various tumors, suggesting that the PD-L1/PD-1 pathway plays an important role in tumor progression (13). This targeted immunotherapy has the potential to improve the prognosis of cancer patients by inhibiting the PD-L1/PD-1 pathway (35-37). Thus, it is imperative to further identify and develop anti-PD-L1/PD-1 therapeutics for TNBC.

Detection of PD-L1. Detection of PD-L1 levels in tumors predicts patient response to anti-PD-L1/PD-1 monoclonal antibody therapy. This allows the screening of selective patients to undergo immunotherapy to reduce unnecessary waste of resources and over-treatment (38). Using the PD-1 monoclonal antibody, pembrolizumab, in patients with non-small cell lung cancer has revealed a $>50 \%$ correlation between the expression of PD-L1 in tumor cells and increased efficacy (39). Immunohistochemistry (IHC) is the most commonly used method for detecting the expression of PD-L1 in tumors. IHC is a simple method that is associated with reduced time for sample processing, low cost, and enhanced visualization. However, in recent years the reliability and reproducibility of this technique have come into question. A disadvantage is with tissue organization (40). Studies have revealed that surgically resected preoperative specimens for tissue biopsy differentially express PD-L1, and biopsy specimens underestimate the expression of PD-L1 as compared to the expression in intraoperative resected specimens (41). PD-L1 levels are affected by focal expression, genetic heterogeneity, and a variety of complex factors within the tumor (42). A routine diagnostic biopsy for measuring PD-L1 expression is highly likely to be a false negative result that biases the sensitivity of PD-L1 targeted therapy (43). Moreover, there is no standardized antibody currently available for the detection of PD-L1. Different investigators use different detection 
antibodies whose combined antigenic epitopes do not match. Thus, the same sample may show opposing results. Owing to these differences, an antibody from an effective clinical trial may not be reliable for other patient populations (44). Finally, setting a threshold for a positive readout is tricky and there is no standard for determining the expression of PD-L1 (45).

Researchers have compared the efficacy of determining PD-L1 expression using IHC and quantitative immunofluorescence (QIF). QIF has been revealed to be more consistent and reproducible than IHC, and the measurement of PD-L1 levels by QIF has been demonstrated to be more objective (44). Detecting the mRNA levels of PD-L1 in tumors is also important. A novel RNA detection technology, RNAScope, has been used to determine the mRNA levels of PD-L1 in 636 patients with stage I-III breast cancer. Surface expression of PD-L1 on circulating tumor cells was successfully detected in patients with hormone receptor-positive, HER-2-negative metastatic breast cancer $(46,47)$. Further developing this technology may prove useful as a non-invasive technique for measuring PD-L1 expression in a liquid biopsy format to screen and treat patients with PD-L1/PD-1 immunotherapy by means of clinical trials in the future.

Regulation of PD-L1. The expression of PD-L1 on the surface of immune cells is relatively constant, whereas PD-L1 expression on the surface of tumor cells is dynamic (48). In addition to the effects of interferon- $\gamma$, PD-L1 expression is also affected by signaling pathways, chemotherapy, radiation therapy, as well as other factors (49). In anaplastic lymphoma kinase (ALK)-positive T-cell lymphoma, the oncogene NPM/ALK was revealed to activate transcription transducer and activator of transcription 3 (STAT3) (50). It has been revealed to bind to the promoter of PD-L1 to upregulate PD-L1 and promote immunosuppression $(50,51)$.

In breast cancer, the inactivation of PTEN or mutation of PI3K leads to PI3K activation, which in turn activates the downstream pathways Akt and mTOR and promotes PD-L1 transcription and protein expression in a ribosomal protein S6 Kinase 1 (S6K1)-dependent manner $(52,53)$. Tumor cells escape immune surveillance and produce immune resistance (54). In melanoma, tumor cells that are tolerant to BRAF gene inhibitors were revealed to promote PD-L1 expression by activating MAPK signaling via the c-Jun and STAT3 pathways (55). Moreover, tumor intervention also affects PD-L1 expression on tumor cells. Using chemotherapeutics, such as doxorubicin, has been revealed to downregulate PD-L1 on the surface of breast cancer cells. The expression of PD-L1 in the nucleus was increased; this may lead to chemoresistance and inhibition of apoptosis of tumor cells. The inhibition of PD-L1, doxorubicin-induced apoptosis was revealed to be increased $(55,56)$. However, chemotherapeutics, such as paclitaxel, were reveealed to upregulate PD-L1 on the surface of ovarian cancer cells via $\mathrm{NF}-\kappa \mathrm{B}$ signaling and inhibition of T-cell function, thereby leading to immune evasion. Combining paclitaxel and PD-L1/PD-1 inhibitors was revealed to prolong patient survival (Fig. 1) (57). In addition, radiation therapy also affects the expression of PD-L1. There is increased surface expression of PD-L1 on metastatic cancer cells that are resistant to radiation therapy, resulting in the depletion of TILs and therapeutic resistance (58). Inhibiting
PD-L1/PD-1 signaling during radiation therapy reverses this phenotype of T-cell depletion and promotes T-cell proliferation $(59,60)$. This indicates that it is necessary to concurrently inhibit PD-L1/PD-1 signaling during chemotherapy and radiation therapy to reduce the immune resistance of tumor cells, enhance the therapeutic effect, and improve the prognosis of patients.

\section{Prognostic significance of PD-1/PD-L1}

PD-L1 is overexpressed in most breast cancers, especially TNBC tissues, as compared to its expression in normal breast tissue (59,61-64). Previous research revealed that PD-L1 was expressed in $45 \%$ of breast cancers and $59 \%$ of TNBCs among 116 breast cancer tissues. Overexpression of PD-L1 has been revealed to be associated with poor prognosis (63), larger tumors, higher tumor grade, estrogen receptor (ER)-negative, progesterone receptor-negative, and HER-2-positive status, cell proliferation, and an increased abundance of TILs in patients with breast cancer $(59,64,65)$. Using high-throughput analysis of 650 breast cancer tissue microarrays, it was revealed that patients with breast cancer and overexpression of PD-L1 had significantly shorter overall survival (OS) (66). Previous research has demonstrated that PD-L1 expression is associated with prolonged recurrence-free survival (62). In patients with TNBC, overexpression of PD-L1 was also associated with metastasis-free survival and specific OS. The higher the expression of PD-L1, the higher the reactivity to chemotherapy (65). The potential of PD-L1 as an independent prognostic factor is unclear. This could be attributed to a variety of factors, such as detection methods and sample heterogeneity; however, it is widely recognized that PD-L1 is associated with factors related to the poor prognosis of breast cancer (42). Tumors overexpressing PD-L1 are often accompanied by the infiltration of PD-1-positive TILs that are associated with shortened OS, indicating a poor prognosis of breast cancer $(67,68)$. This further highlights the need for utilizing PD-L1/PD-1 signaling in developing efficacious therapeutics for the treatment of TNBC.

\section{Significance of targeting PD-1/PD-L1 monoclonal antibody}

Binding of PD-1 to PD-L1 or PD-L2 activates PD-1 signaling (69). PD-L1 is key for the proliferation of tumor cells during antitumor immunity. Notably, tumor immunogenicity is weakened significantly and this promotes the escape of the tumor cells from immune surveillance and response. PD-L1/PD-1 plays a crucial positive stimulating role in tumor invasion and metastasis (70). Blocking the PD-L1/PD-1 interaction reduces the inhibition of innate immunity and promotes tumor-specific T-cell activation (28).

Application of PD-L1/PD-1 inhibitor. Drugs targeted to block PD-1 signaling have exhibited sustained clinical activity in numerous advanced solid tumors (Fig. 2) (71). The use of the monoclonal antibody BMS-936559 to block PD-L1 in 160 patients with advanced solid tumors during phase I clinical trials revealed an objective response rate of $6-17 \%$ (72). The objective response rates of using the PD-1-targeting antibody, nivolumab, in the treatment of advanced non-small cell lung 


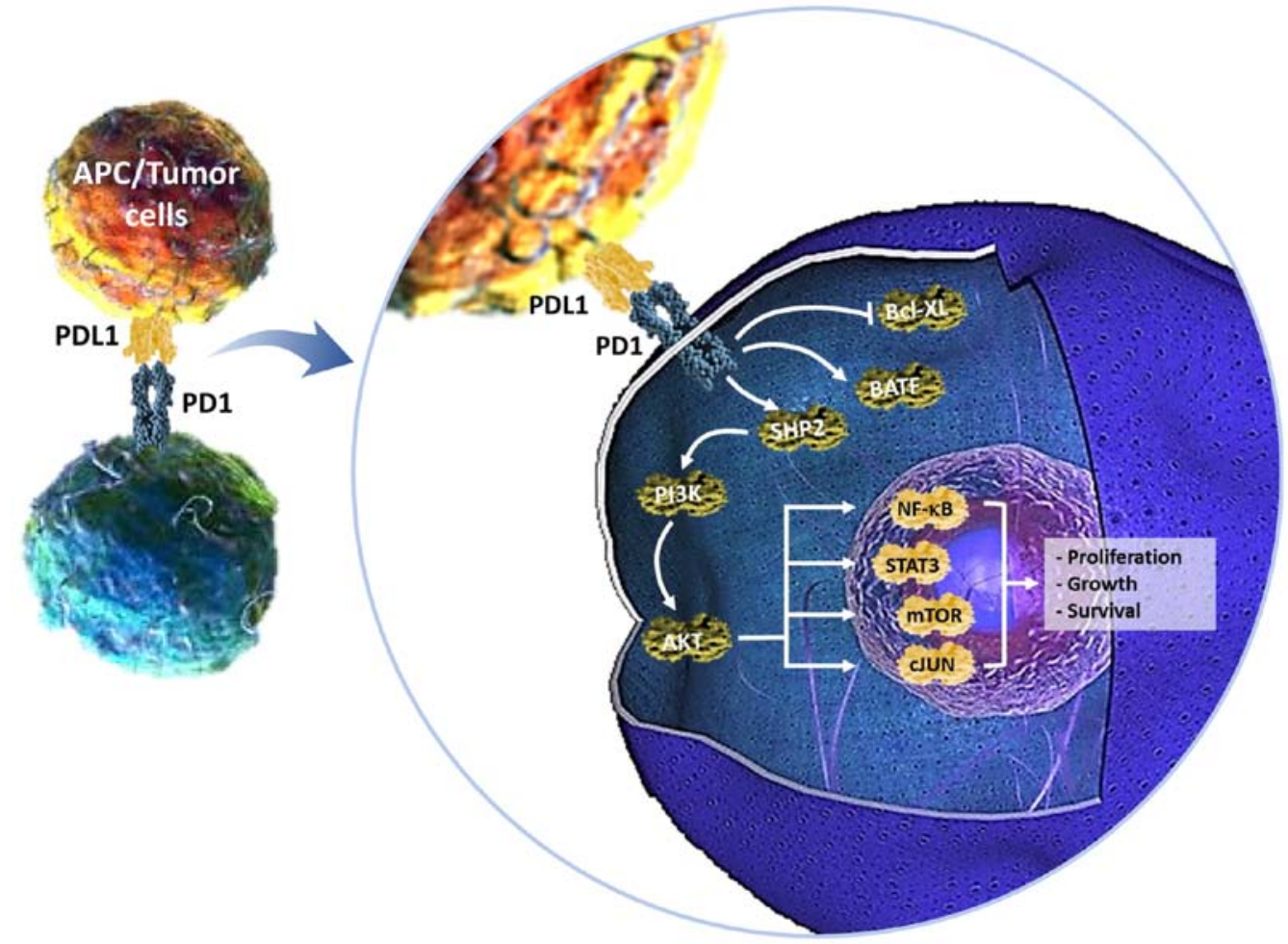

Figure 1. Mechanism of how PD-1 inhibits T-cell receptor signaling is under focus of investigation. APC, antigen-presenting cell; mTOR, mechanistic target of rapamycin; NF- $\mathrm{BB}$, nuclear factor- $\mathrm{\kappa B}$; PD-1, programmed cell death protein 1; PD-L1, programmed death-ligand 1; PTEN, phosphatase and tensin homolog; SHP, Src homology region 2 domain-containing phosphatise.

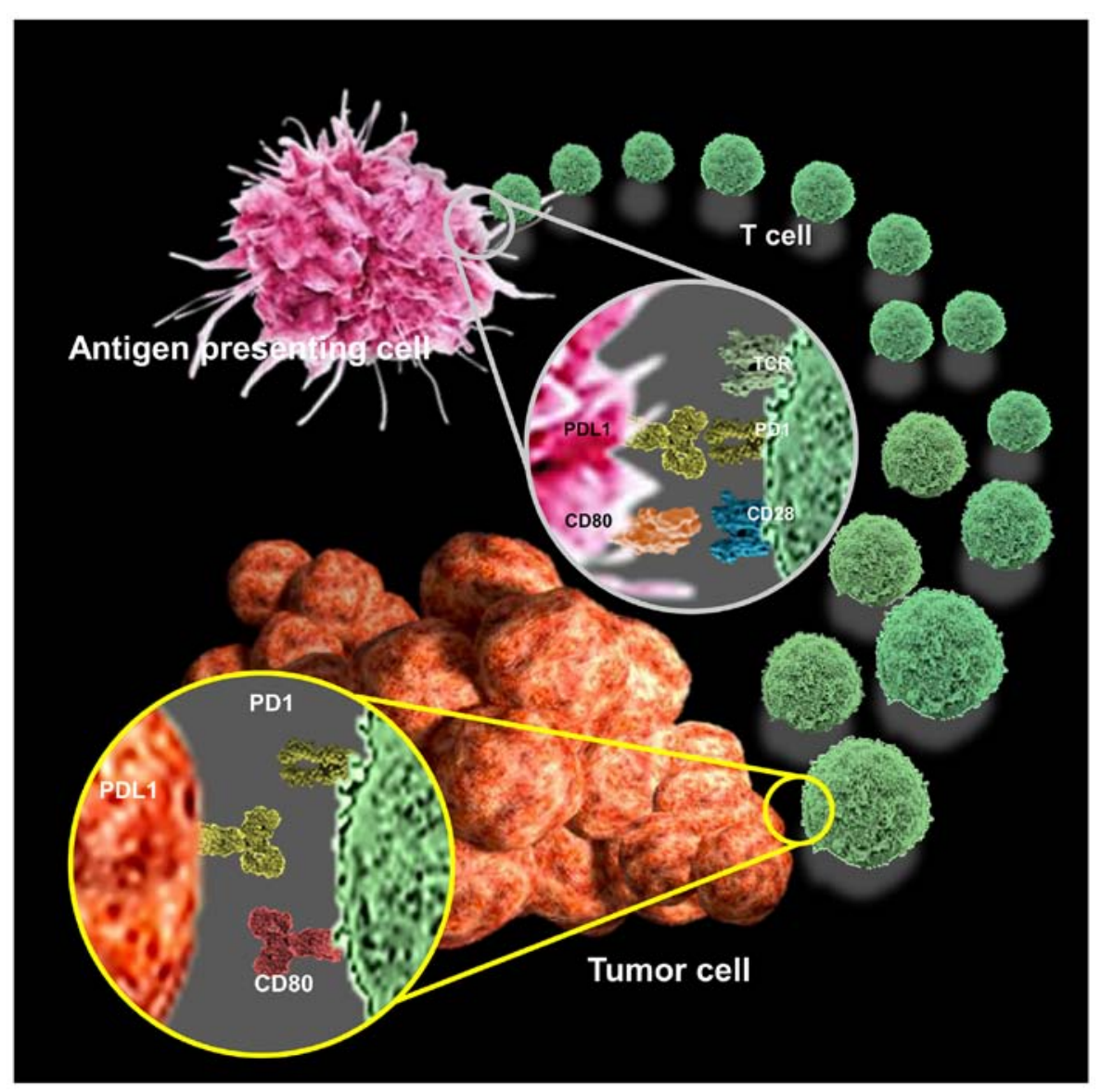

Figure 2. Mechanism of PD-1 receptor and PD-L1/L2 inhibitors-mediated cancer immunotherapy. The interaction between tumor-intrinsic PD-1 and PD-L1 inhibits tumor progression, but the treatment by anti-PD-1 disrupts this inhibitory signaling and promotes tumor progression. This process represents an adverse effect of anti-PD-1 therapy for activating antitumor immunity. PD-1, programmed cell death protein 1; PD-L1, programmed death-ligand 1. 
cancer, bladder cancer, chondrosarcoma, melanoma, and renal cell carcinoma were revealed to be 18,28 and $27 \%$, respectively (35,73-76). The KEYNOTE-028 trial evaluated the safety and efficacy of pembrolizumab in metastatic $\mathrm{ER}^{+}$breast cancer. PD-L1 was expressed in $19 \%$ of the samples and the efficacy of treatment in 25 evaluable patients was analyzed. The total effective rate was $12 \%$ (77). Nanda et al evaluated the safety and efficacy of pembrolizumab in 32 patients with advanced TNBC who were positive for PD-L1. Most patients received 1-3 cycles of chemotherapy before being administered pembrolizumab. A minority of patients $(21.9 \%)$ were administered five or more cycles of chemotherapy. Efficacy analysis of 27 evaluable patients revealed a total effective rate of $18.5 \%$, including one patient with complete response and two patients exhibiting partial remission. Most of the adverse reactions were grade 1-2, including joint pain, fatigue, muscle pain, and nausea (78). The ORR for 21 PD-L1+ patients with metastatic triple-negative breast cancer evaluable for efficacy was $19 \%$, including 2 CRs and 2 PRs; 3 of 4 of these responses were ongoing at the time of data cutoff. The JAVELIN study tested the efficacy of the anti-PD-L1 antibody, avelumab, on patients with a variety of all breast cancer types, regardless of the extent of PD-L1 expression. ER ${ }^{+} / \mathrm{HER} 2^{-}$patients in the TNBC group (58 cases) had a response rate of $8.6 \%$. Among the 72 patients and $26 \mathrm{HER}^{+}$patients, the response rates were 2.8 and $3.8 \%$, respectively. Preliminary results have revealed that PD-L1-positive tumors exhibit a higher response rate (77).

PD-L1/PD-1 inhibitor combined with anticancer drugs. TILs are an independent prognostic factor for improved OS, reduced distant recurrence, and increased metastasis-free survival in newly diagnosed patients with TNBC (79). Retrospective analysis of several large clinical trials and randomized neoadjuvant studies have demonstrated that high abundance of TILs in tumors have predictive effects on neoadjuvant chemotherapy for pCR or increased disease-free survival and OS (80). In addition, a recent retrospective analysis confirmed that the presence of TILs in patients with residual lesions after neoadjuvant chemotherapy can predict patient outcomes (81). Previous research has confirmed a significant association between activated Ras-MAPK signaling and low abundance of TILs in TNBC patients. Activated MEK inhibits IFN- $\gamma$-induced antigen presentation. This is because the Ras/MAPK pathway initiates immune evasion, and inhibition of MEK signaling upregulates PD-L1 expression on TNBC cells (82). Combining MEK and PD-L1/PD-1 inhibitors was revealed to enhance the antitumor immune response in a mouse model of breast cancer (82). Sagiv-Barfi et al used ibrutinib in combination with an anti-PD-L1 antibody, ibrutinib, to treat mice with TNBC that were not intrinsically sensitive to ibrutinib. The combination was determined to significantly delay tumor growth and improve survival relative to drug administration alone (83).

Concurrent vaccination with PD-L1/PD-1 inhibitor. For patients with metastatic breast cancer, monotherapy, including vaccination or monoclonal antibodies against immune checkpoints, such as anti-PD-1 or PD-L1, may not be sufficient to eradicate the lesion (84). Vaccines stimulate antigen-specific $\mathrm{T}$ cells after combination therapy. Monoclonal antibodies targeting the immune checkpoint allow antigen-specific
$\mathrm{T}$ cells to proliferate. This approach may enhance antitumor immune responses, thereby increasing tumor cell death and improving patient outcomes (85). IHC of biopsy specimens from 42 patients evaluated the expression of PD-L1 on the surface of tumor cells. Notably, none of the 17 PD-L1-negative patients had an objective response to PD-1 therapy. Despite the small patient cohort, this data suggests that PD-L1 expression on the surface of tumor cells may be a biomarker for the efficacy of anti-PD-1 therapy (35). Melanoma patients and PD-L1-positive tumor patients treated with nivolumab demonstrated higher objective response rates, longer progression-free survival, and improved OS. Ongoing and future studies on the efficacy of nivolumab and other anti-PD-L1/PD-1 drugs will help confirm whether PD-L1 is a potent biomarker for predicting responsiveness to anti-PD-L1/PD-1 therapy (85). Chatterjee et al have revealed that the non-invasive detection of PD-L1 expression in the tumor microenvironment will enable the appropriate administration of monoclonal antibodies targeting PD-L1/PD-1 (86).

\section{Anti-PD-L1 therapy for breast cancer}

Overexpression of PD-L1 and PD-1 and abundance of TILs suggest that PD-L1/PD-1 are reliable candidates for PD-L1/PD-1 immunotherapy $(35,38,72,87,88)$. The current treatment methods for breast cancer primarily include surgery, radiation therapy, chemotherapy, endocrine therapy, and targeted therapy. There are no specific and effective treatment options for TNBC patients owing to the lack specific targets, resulting in unsatisfactory disease prognosis. To that extent, the emergence of PD-L1 as a novel target is an exciting avenue in TNBC treatment $(58,70)$.

A phase 1 clinical trial comprising patients with metastatic TNBC was reported in 2015; the monoclonal antibody, atezolizumab, targeting PD-L1 was demonstrated to be safe, tolerable, and consistently exhibited antitumor effects (89). In addition to the PD-L1/PD-1 antibody, clinical trials have used other immunological targets to enhance its antitumor effects, such as the CTLA-4 monoclonal antibody ipilimumab (90). CTLA-4 monoclonal antibodies block negative co-stimulatory signaling, promote the activation and proliferation of tumor-specific T cells, and prevent T-cell disability. PD-1 and PD-L1-targeting monoclonal antibodies reverse the immunosuppressive state of the tumor microenvironment by inhibiting the PD-L1/PD-1 axis. Compared with the CTL-4 monoclonal antibody, the PD-1 and PD-L1 monoclonal antibodies are associated with reduced adverse reactions, improved tolerance, and safety. Thus, PD-L1 antibodies are also used in combination with chemotherapeutics, such as atezolizumab in combination with white protein and abraxane or MEDI4736 (durvalumab) in combination with ibrutinib, in patients with TNBC (91). Several clinical trials are underway to evaluate the efficacy of PD-L1/PD-1 monoclonal antibodies in treating TNBC (Table I).

\section{Future perspectives}

The past few decades have seen significant progress in the treatment of breast cancer. This has been associated with decreased rates of mortality rate in patients with breast 
Table I. Immunotherapeutic agents (anti-PD-L1) in clinical trials.

\begin{tabular}{lllc}
\hline No. & \multicolumn{1}{c}{ Drugs } & Target & Phase \\
\hline NCT02622074 & Pembrolizumab & PD-1 & I \\
NCT02795429 & INC280 & PD-1 & I \\
NCT02530125 & Pidilizumab & PD-1 & II \\
NCT02644369 & Pembrolizumab & PD-1 & II \\
NCT02447003 & Pembrolizumab & PD-1 & II \\
NCT02555657 & Pembrolizumab & PD-1 & III \\
NCT02967692 & Dabrafenib, Trametinib, & PD-1 & III \\
& LCL161 & & \\
NCT02403271 & MEDI4736 & PD-L1 & I, II \\
NCT02484404 & MEDI4736 & PD-L1 & I, II \\
NCT02530489 & Atezolizumab & PD-L1 & II \\
NCT02724878 & Bevacizumab & PD-L1 & II \\
NCT02620280 & Atezolizumab & PD-L1 & III \\
NCT02425891 & Atezolizumab & PD-L1 & III \\
NCT02008227 & Docetaxel & PD-L1 & III \\
NCT02302807 & Docetaxel, Paclitaxel, & PD-L1 & III \\
& Vinflunine & & \\
& & &
\end{tabular}

PD-L1, programmed death-ligand 1; PD-1, programmed cell death protein 1 .

cancer and improved quality of life. However, the prognosis for most patients with TNBC remains poor (92). Exploring TNBC-specific therapeutic targets is key to prolonging patient survival and further improving quality of life. PD-L1/PD-1 signaling is currently a research hotspot for tumor immunotherapy (84). Although PD-L1/PD-1 immunotargeting drugs have not been approved by the FDA for the treatment of TNBC, clinical trials have revealed encouraging results. Thus, PD-L1/PD-1 immunotargeting drugs are expected to be used in the treatment of patients with TNBC in the near future (92).

Despite this, several issues remain worthy of further discussion: i) PD-L1 detection methods, and outcome judgment criteria need to be further improved to accurately determine the expression of PD-L1 and select patients to avoid over- or ineffective treatment. ii) Recently, new members of the B7 family other than PD-L1/PD-1 have been discovered and may form novel targets for tumor immunotherapy. For example, PD-L2 is also expressed in TNBC; thus, anti-PD-1 antibody may be ineffective for PD-L1-negative patients. The reason why the expressed patient is effective; B7-H3 and B7-H4 have immunosuppressive roles and are expressed in tumor cells. Thus, they are excellent novel candidates to be used in tumor immunotherapy; however, their physiological functions remain to be studied (93). iii) The accuracy and adverse reactions of PD-L1/PD-1 immunotargeting therapy need to monitored. It is important to target PD-L1/PD-1-specific drugs to tumor cells during treatment and reduce the impact on autoimmune function. The growing research on these candidates will soon establish immunotherapy as an indispensable method for the comprehensive treatment of cancers.

\section{Acknowledgements}

Not applicable.

\section{Funding}

This work was supported by grants from the Ministry of Science and Technology (grant nos. MOST 106-2314-B-442-001-MY3, MOST 109-2314-B-442-001 and MOST 109-2314-B-075B-002), the National Health Research Institutes (grant no. NHRI109BCCO-MF-202015-01) and Show Chwan Memorial Hospital, Taiwan (grant nos. SRD-109023 and RD107063).

\section{Availability of data and materials}

Data sharing is not applicable to this article, as no datasets were generated or analyzed during the current study.

\section{Authors' contributions}

CJL, LTL, MFH and PYC conceived the study. CJL and LTL wrote the study. CJL and PYC reviewed and edited the study. PYC supervised the study. All authors reviewed the final version of the manuscript.

\section{Ethics approval and consent to participate}

Not applicable.

\section{Patient consent for publication}

Not applicable.

\section{Competing interests}

The authors declare that they have no competing interests.

\section{References}

1. Seyfried TN and Huysentruyt LC: On the origin of cancer metastasis. Crit Rev Oncog 18: 43-73, 2013.

2. Harbeck N, Penault-Llorca F, Cortes J, Gnant M, Houssami N, Poortmans P, Ruddy K, Tsang $\mathbf{J}$ and Cardoso F: Breast cancer. Nat Rev Dis Primers 5: 66, 2019.

3. Nowicki TS, Hu-Lieskovan S and Ribas A: Mechanisms of resistance to PD-1 and PD-L1 blockade. Cancer J 24: 47-53, 2018.

4. Ju X, Zhang H, Zhou Z and Wang Q: Regulation of PD-L1 expression in cancer and clinical implications in immunotherapy. Am J Cancer Res 10: 1-11, 2020.

5. Feng Y, Spezia M, Huang S, Yuan C, Zeng Z, Zhang L, Ji X, Liu W, Huang B, Luo W, et al: Breast cancer development and progression: Risk factors, cancer stem cells, signaling pathways, genomics, and molecular pathogenesis. Genes Dis 5: 77-106, 2018.

6. Zhang Z, Zhou L, Xie N, Nice EC, Zhang T, Cui Y and Huang C: Overcoming cancer therapeutic bottleneck by drug repurposing. Signal Transduct Target Ther 5: 113, 2020.

7. Li CJ, Liao WT, Wu MY and Chu PY: New insights into the role of autophagy in tumor immune microenvironment. Int J Mol Sci 18: 1566, 2017.

8. Schreiber RD, Old LJ and Smyth MJ: Cancer immunoediting: Integrating immunity's roles in cancer suppression and promotion. Science 331: 1565-1570, 2011.

9. Couzin-Frankel J: Breakthrough of the year 2013. Cancer immunotherapy. Science 342: 1432-1433, 2013.

10. Dong Y, Sun Q and Zhang X: PD-1 and its ligands are important immune checkpoints in cancer. Oncotarget 8: 2171-2186, 2017. 
11. Matsushita $\mathbf{M}$ and Kawaguchi M: Immunomodulatory effects of drugs for effective cancer immunotherapy. J Oncol 2018: 8653489, 2018

12. Lin SC, Chu PY, Liao WT, Wu MY, Tsui KH, Lin LT, Huang $\mathrm{CH}$, Chen LL and Li CJ: Glycyrrhizic acid induces human MDA-MB-231 breast cancer cell death and autophagy via the ROS-mitochondrial pathway. Oncol Rep 39: 703-710, 2018

13. Song Y, He L, Wang Y, Wu Q and Huang W: Molecularly targeted therapy and immunotherapy for hormone receptorpositive/human epidermal growth factor receptor 2-negative advanced breast cancer (review). Oncol Rep 44: 3-13, 2020.

14. Chew V, Toh HC and Abastado JP: Immune microenvironment in tumor progression: Characteristics and challenges for therapy. J Oncol 2012: 608406, 2012.

15. Chen SN, Chang R, Lin LT, Chern CU, Tsai HW, Wen ZH, Li YH, Li CJ and Tsui KH: MicroRNA in ovarian cancer: Biology, pathogenesis, and therapeutic opportunities. Int J Environ Res Public Health 16: 1510, 2019.

16. Li YT, Lee WL and Tsui KH: Endometrial thickness still presents a best reference to predict endometrial cancer. Taiwan J Obstet Gynecol 55: 148-149, 2016.

17. Wu MY, Yiang GT, Cheng PW, Chu PY and Li CJ: Molecular targets in hepatocarcinogenesis and implications for therapy. J Clin Med 7: 213, 2018

18. Xia A, Zhang Y, Xu J, Yin T and Lu XJ: T cell dysfunction in cancer immunity and immunotherapy. Front Immunol 10: 1719, 2019.

19. Mahoney KM, Rennert PD and Freeman GJ: Combination cancer immunotherapy and new immunomodulatory targets. Nat Rev Drug Discov 14: 561-584, 2015.

20. Roncati L: Microsatellite instability predicts response to anti-PD1 immunotherapy in metastatic melanoma. Acta Dermatovenerol Croat 26: 341-343, 2018

21. Ribas A: Tumor immunotherapy directed at PD-1. N Engl J Med 366: 2517-2519, 2012

22. Okazaki T and Honjo T: The PD-1-PD-L pathway in immunological tolerance. Trends Immunol 27: 195-201, 2006.

23. Sun $H$, Sun $C$ and Xiao W: Expression regulation of co-inhibitory molecules on human natural killer cells in response to cytokine stimulations. Cytokine 65: 33-41, 2014.

24. Hirsch I, Janovec V, Stranska R and Bendriss-Vermare N: Cross talk between inhibitory immunoreceptor tyrosine-based activation motif-signaling and toll-like receptor pathways in macrophages and dendritic cells. Front Immunol 8: 394, 2017.

25. Pedoeem A, Azoulay-Alfaguter I, Strazza M, Silverman GJ and Mor A: Programmed death-1 pathway in cancer and autoimmunity. Clin Immunol 153: 145-152, 2014.

26. Okazaki T and Honjo T: PD-1 and PD-1 ligands: From discovery to clinical application. Int Immunol 19: 813-824, 2007.

27. Nawaf MG, Ulvmar MH, Withers DR, McConnell FM Gaspal FM, Webb GJ, Jones ND, Yagita H, Allison JP and Lane PJ: Concurrent OX40 and CD30 ligand blockade abrogates the CD4-driven autoimmunity associated with CTLA4 and PD1 blockade while preserving excellent anti-CD8 tumor immunity. J Immunol 199: 974-981, 2017.

28. Homet Moreno B and Ribas A: Anti-programmed cell death protein-1/ligand-1 therapy in different cancers. Br J Cancer 112: $1421-1427,2015$

29. Chiu HC, Li CJ, Yiang GT, Tsai AP and Wu MY: Epithelial to mesenchymal transition and cell biology of molecular regulation in endometrial carcinogenesis. J Clin Med 8: 439, 2019.

30. Tsui KH, Chiang AJ and Yu KJ: Urgent surgical intervention for ruptured ovarian endometrioma. Taiwan J Obstet Gynecol 51: $328,2012$.

31. Dai S, Jia R, Zhang X, Fang Q and Huang L: The PD-1/PD-Ls pathway and autoimmune diseases. Cell Immunol 290: 72-79, 2014

32. Joshi S and Durden DL: Combinatorial approach to improve cancer immunotherapy: Rational drug design strategy to simultaneously hit multiple targets to kill tumor cells and to activate the immune system. J Oncol 2019: 5245034, 2019.

33. Zhang M, Yang J, Zhou J, Gao W, Zhang Y, Lin Y, Wang $\mathrm{H}$, Ruan Z and Ni B: Prognostic Values of CD $38^{+} \mathrm{CD}_{101}{ }^{+} \mathrm{PD} 1^{+} \mathrm{CD} 8{ }^{+}$ $\mathrm{T}$ cells in pancreatic cancer. Immunol Invest 48: 466-479, 2019.

34. Nguyen LT and Ohashi PS: Clinical blockade of PD1 and LAG3-potential mechanisms of action. Nat Rev Immunol 15 45-56, 2015.

35. Topalian SL, Hodi FS, Brahmer JR, Gettinger SN, Smith DC, McDermott DF, Powderly JD, Carvajal RD, Sosman JA, Atkins MB, et al: Safety, activity, and immune correlates of anti-PD-1 antibody in cancer. N Engl J Med 366: 2443-2454, 2012.
36. Reiss KA, Forde PM and Brahmer JR: Harnessing the power of the immune system via blockade of PD-1 and PD-L1: A promising new anticancer strategy. Immunotherapy 6: 459-475, 2014.

37. Powles T, Eder JP, Fine GD, Braiteh FS, Loriot Y, Cruz C, Bellmunt J, Burris HA, Petrylak DP, Teng SL, et al: MPDL3280A (anti-PD-L1) treatment leads to clinical activity in metastatic bladder cancer. Nature 515: 558-562, 2014.

38. Taube JM, Klein A, Brahmer JR, Xu H, Pan X, Kim JH, Chen L, Pardoll DM, Topalian SL and Anders R: Association of PD-1, $\mathrm{PD}-1$ ligands, and other features of the tumor immune microenvironment with response to anti-PD-1 therapy. Clin Cancer Res 20: 5064-5074, 2014

39. Garon EB, Rizvi NA, Hui R, Leighl N, Balmanoukian AS, Eder JP, Patnaik A, Aggarwal C, Gubens M, Horn L, et al: Pembrolizumab for the treatment of non-small-cell lung cancer. N Engl J Med 372: 2018-2028, 2015.

40. Koppel C, Schwellenbach H, Zielinski D, Eckstein S, Martin-Ortega M, D'Arrigo C, Schildhaus HU, Rüschoff J and Jasani B: Optimization and validation of PD-L1 immunohistochemistry staining protocols using the antibody clone $28-8$ on different staining platforms. Mod Pathol 31: 1630-1644, 2018.

41. Ilie M, Long-Mira E, Bence C, Butori C, Lassalle S, Bouhlel L, Fazzalari L, Zahaf K, Lalvée S, Washetine K, et al: Comparative study of the PD-L1 status between surgically resected specimens and matched biopsies of NSCLC patients reveal major discordances: A potential issue for anti-PD-L1 therapeutic strategies. Ann Oncol 27: 147-153, 2016

42. Wu Z, Zhang L, Peng J, Xu S, Zhou L, Lin Y, Wang Y, Lu J, Yin $\mathrm{W}$ and Lu J: Predictive and prognostic value of PDL1 protein expression in breast cancer patients in neoadjuvant setting. Cancer Biol Ther 20: 941-947, 2019.

43. Gerlinger M, Rowan AJ,Horswell S,Math M,Larkin J,Endesfelder D, Gronroos E, Martinez P, Matthews N, Stewart A, et al: Intratumor heterogeneity and branched evolution revealed by multiregion sequencing. N Engl J Med 366: 883-892, 2012.

44. McLaughlin J, Han G, Schalper KA, Carvajal-Hausdorf D, Pelekanou V, Rehman J, Velcheti V, Herbst R, LoRusso P and Rimm DL: Quantitative assessment of the heterogeneity of PD-L1 expression in non-small-cell lung cancer. JAMA Oncol 2: 46-54, 2016.

45. Ilie M, Hofman V, Dietel M, Soria JC and Hofman P: Assessment of the PD-L1 status by immunohistochemistry: Challenges and perspectives for therapeutic strategies in lung cancer patients. Virchows Arch 468: 511-525, 2016.

46. Schalper KA, Velcheti V, Carvajal D, Wimberly H, Brown J, Pusztai L and Rimm DL: In situ tumor PD-L1 mRNA expression is associated with increased TILs and better outcome in breast carcinomas. Clin Cancer Res 20: 2773-2782, 2014.

47. Mazel M, Jacot W, Pantel K, Bartkowiak K, Topart D, Cayrefourcq L, Rossille D, Maudelonde T, Fest T and Alix-Panabières C: Frequent expression of PD-L1 on circulating breast cancer cells. Mol Oncol 9: 1773-1782, 2015.

48. Yue C, Jiang Y, Li P, Wang Y, Xue J, Li N, Li D, Wang R, Dang Y, $\mathrm{Hu} \mathrm{Z}$, et al: Dynamic change of PD-L1 expression on circulating tumor cells in advanced solid tumor patients undergoing PD-1 blockade therapy. Oncoimmunology 7: e1438111, 2018.

49. Acheampong E, Spencer I, Lin W, Ziman M, Millward M and Gray E: Is the blood an alternative for programmed cell death ligand 1 assessment in non-small cell lung cancer? Cancers (Basel) 11: 920, 2019.

50. Marzec M,Zhang Q, Goradia A, Raghunath PN,Liu X,Paessler M, Wang HY, Wysocka M, Cheng M, Ruggeri BA and Wasik MA: Oncogenic kinase NPM/ALK induces through STAT3 expression of immunosuppressive protein CD274 (PD-L1, B7-H1). Proc Natl Acad Sci USA 105: 20852-20857, 2008.

51. Tang J, Yu JX, Hubbard-Lucey VM, Neftelinov ST, Hodge JP and Lin Y: Trial watch: The clinical trial landscape for PD1/PDL1 immune checkpoint inhibitors. Nat Rev Drug Discov 17: 854-855, 2018.

52. Holz MK: The role of S6K1 in ER-positive breast cancer. Cell Cycle 11: 3159-3165, 2012.

53. Sridharan $\mathrm{S}$ and Basu A: Distinct roles of mTOR targets S6K1 and S6K2 in breast cancer. Int J Mol Sci 21: 1199, 2020.

54. Crane CA, Panner A, Murray JC, Wilson SP, Xu H, Chen L, Simko JP, Waldman FM, Pieper RO and Parsa AT: PI(3) kinase is associated with a mechanism of immunoresistance in breast and prostate cancer. Oncogene 28: 306-312, 2009.

55. Jiang X, Zhou J, Giobbie-Hurder A, Wargo J and Hodi FS: The activation of MAPK in melanoma cells resistant to BRAF inhibition promotes PD-L1 expression that is reversible by MEK and PI3K inhibition. Clin Cancer Res 19: 598-609, 2013. 
56. Ghebeh H, Lehe C, Barhoush E, Al-Romaih K, Tulbah A, Al-Alwan M, Hendrayani SF, Manogaran P, Alaiya A, Al-Tweigeri $\mathrm{T}$, et al: Doxorubicin downregulates cell surface B7-H1 expression and upregulates its nuclear expression in breast cancer cells: Role of B7-H1 as an anti-apoptotic molecule. Breast Cancer Res 12: R48, 2010.

57. Peng J, Hamanishi J, Matsumura N, Abiko K, Murat K, Baba T, Yamaguchi K, Horikawa N, Hosoe Y, Murphy SK, et al: Chemotherapy induces programmed cell death-ligand 1 overexpression via the nuclear factor- $\kappa \mathrm{B}$ to foster an immunosuppressive tumor microenvironment in ovarian cancer. Cancer Res 75: 5034-5045, 2015.

58. Uhercik M, Sanders AJ, Owen S, Davies EL, Sharma AK, Jiang WG and Mokbel K: Clinical significance of PD1 and PDL1 in human breast cancer. Anticancer Res 37: 4249-4254, 2017.

59. Twyman-Saint Victor C, Rech AJ, Maity A, Rengan R, Pauken KE Stelekati E, Benci JL, Xu B, Dada H, Odorizzi PM, et al: Radiation and dual checkpoint blockade activate non-redundant immune mechanisms in cancer. Nature 520: 373-377, 2015.

60. Lv S, Wang S, Qiao G, Wang X, Zhou X, Yan F, Li Y, Wang S, Morse MA, Hobeika A, et al: Functional $\mathrm{CD}^{+}{ }^{+} \mathrm{CD} 8^{+} \mathrm{PD} 1^{-} \mathrm{T}$ cell accumulation and PD-L1 expression increases during tumor invasion in DCIS of the breast. Clin Breast Cancer 19: e617-e623, 2019.

61. Beckers RK, Selinger CI, Vilain R, Madore J, Wilmott JS, Harvey K, Holliday A, Cooper CL, Robbins E, Gillett D, et al: Programmed death ligand 1 expression in triple-negative breast cancer is associated with tumour-infiltrating lymphocytes and improved outcome. Histopathology 69: 25-34, 2016.

62. Mittendorf EA, Philips AV, Meric-Bernstam F, Qiao N, Wu Y, Harrington S, Su X, Wang Y, Gonzalez-Angulo AM, Akcakanat A, et al: PD-L1 expression in triple-negative breast cancer. Cancer Immunol Res 2: 361-370, 2014.

63. Gatalica Z, Snyder C, Maney T, Ghazalpour A, Holterman DA, Xiao N, Overberg P, Rose I, Basu GD, Vranic S, et al: Programmed cell death 1 (PD-1) and its ligand (PD-L1) in common cancers and their correlation with molecular cancer type. Cancer Epidemiol Biomarkers Prev 23: 2965-2970, 2014.

64. Bertucci F, Finetti P, Colpaert C, Mamessier E, Parizel M, Dirix L, Viens P, Birnbaum D and van Laere S: PDL1 expression in inflammatory breast cancer is frequent and predicts for the pathological response to chemotherapy. Oncotarget 6 : 13506-13519, 2015.

65. Sabatier R, Finetti P, Mamessier E, Adelaide J, Chaffanet M, Ali HR, Viens P, Caldas C, Birnbaum D and Bertucci F: Prognostic and predictive value of PDL1 expression in breast cancer. Oncotarget 6: 5449-5464, 2015.

66. Muenst S, Schaerli AR, Gao F, Däster S, Trella E, Droeser RA, Muraro MG, Zajac P, Zanetti R, Gillanders WE, et al: Expression of programmed death ligand 1 (PD-L1) is associated with poor prognosis in human breast cancer. Breast Cancer Res Treat 146: 15-24, 2014.

67. Muenst S, Soysal SD, Gao F, Obermann EC, Oertli D and Gillanders WE: The presence of programmed death 1 (PD-1)-positive tumor-infiltrating lymphocytes is associated with poor prognosis in human breast cancer. Breast Cancer Res Treat 139: 667-676, 2013

68. Sun S, Fei X, Mao Y, Wang X, Garfield DH, Huang O, Wang J, Yuan F, Sun L, Yu Q, et al: PD-1(+) immune cell infiltration inversely correlates with survival of operable breast cancer patients. Cancer Immunol Immunother 63: 395-406, 2014.

69. Pardoll DM: The blockade of immune checkpoints in cancer immunotherapy. Nat Rev Cancer 12: 252-264, 2012.

70. Hasan A, Ghebeh H, Lehe C, Ahmad R and Dermime S Therapeutic targeting of B7-H1 in breast cancer. Expert Opin Ther Targets 15: 1211-1225, 2011.

71. Lipson EJ, Forde PM, Hammers HJ, Emens LA, Taube JM and Topalian SL: Antagonists of PD-1 and PD-L1 in cancer treatment. Semin Oncol 42: 587-600, 2015.

72. Brahmer JR, Tykodi SS, Chow LQ, Hwu WJ, Topalian SL, Hwu P, Drake CG, Camacho LH, Kauh J, Odunsi K, et al: Safety and activity of anti-PD-L1 antibody in patients with advanced cancer. N Engl J Med 366: 2455-2465, 2012.
73. Wang Y, Zhang X, Yang L, Xue J and Hu G: Blockade of CCL2 enhances immunotherapeutic effect of anti-PD1 in lung cancer. J Bone Oncol 11: 27-32, 2018

74. Stenehjem DD, Tran D, Nkrumah MA and Gupta S: PD1/PDL1 inhibitors for the treatment of advanced urothelial bladder cancer. Onco Targets Ther 11: 5973-5989, 2018

75. Wagner MJ, Ricciotti RW, Mantilla J, Loggers ET, Pollack SM and Cranmer LD: Response to PD1 inhibition in conventional chondrosarcoma. J Immunother Cancer 6: 94, 2018.

76. Neubert NJ, Schmittnaegel M, Bordry N, Nassiri S, Wald N, Martignier C, Tillé L, Homicsko K, Damsky W, Maby-El Hajjami $\mathrm{H}$, et al: $\mathrm{T}$ cell-induced CSF1 promotes melanoma resistance to PD1 blockade. Sci Transl Med 10: eaan3311, 2018.

77. Pusztai L, Karn T, Safonov A, Abu-Khalaf MM and Bianchini G: New strategies in breast cancer: Immunotherapy. Clin Cancer Res 22: 2105-2110, 2016.

78. Nanda R, Chow LQM, Dees EC, Berger R, Gupta S, Geva R, Pusztai L, Pathiraja K, Aktan G, Cheng JD, et al: Pembrolizumab in patients with advanced triple-negative breast cancer: phase $\mathrm{Ib}$ KEYNOTE-012 study. J Clin Oncol 34: 2460-2467, 2016.

79. Emens LA: Breast cancer immunobiology driving immunotherapy: Vaccines and immune checkpoint blockade. Expert Rev Anticancer Ther 12: 1597-1611, 2012.

80. Badr NM, Berditchevski F and Shaaban AM: The immune microenvironment in breast carcinoma: Predictive and prognostic role in the neoadjuvant setting. Pathobiology 87: 61-74, 2020.

81. Tan W, Yang M, Yang H, Zhou F and Shen W: Predicting the response to neoadjuvant therapy for early-stage breast cancer: Tumor-, blood-, and imaging-related biomarkers. Cancer Manag Res 10: 4333-4347, 2018.

82. Loi S, Dushyanthen S, Beavis PA, Salgado R, Denkert C, Savas P, Combs S, Rimm DL, Giltnane JM, Estrada MV, et al: RAS/MAPK activation is associated with reduced tumor-infiltrating lymphocytes in triple-negative breast cancer: Therapeutic cooperation between MEK and PD-1/PD-L1 immune checkpoint inhibitors. Clin Cancer Res 22: 1499-1509, 2016.

83. Sagiv-Barfi I, Kohrt HE, Czerwinski DK, Ng PP, Chang BY and Levy R: Therapeutic antitumor immunity by checkpoint blockade is enhanced by ibrutinib, an inhibitor of both BTK and ITK. Proc Natl Acad Sci USA 112: E966-E972, 2015.

84. Garcia-Aranda M and Redondo M: Immunotherapy: A challenge of breast cancer treatment. Cancers (Basel) 11: 1822, 2019.

85. Chawla A, Philips AV, Alatrash G and Mittendorf E: Immune checkpoints: A therapeutic target in triple negative breast cancer. Oncoimmunology 3: e28325, 2014

86. Chatterjee S, Lesniak WG, Gabrielson M, Lisok A, Wharram B, Sysa-Shah P, Azad BB, Pomper MG and Nimmagadda S: A humanized antibody for imaging immune checkpoint ligand PD-L1 expression in tumors. Oncotarget 7: 10215-10227, 2016.

87. Herbst RS, Soria JC, Kowanetz M, Fine GD, Hamid O, Gordon MS Sosman JA, McDermott DF, Powderly JD, Gettinger SN, et al: Predictive correlates of response to the anti-PD-L1 antibody MPDL3280A in cancer patients. Nature 515: 563-567, 2014.

88. Tumeh PC, Harview CL, Yearley JH, Shintaku IP, Taylor EJ, Robert L, Chmielowski B, Spasic M, Henry G, Ciobanu V, et al: PD-1 blockade induces responses by inhibiting adaptive immune resistance. Nature 515: 568-571, 2014.

89. Gibson J: Anti-PD-L1 for metastatic triple-negative breast cancer. Lancet Oncol 16: e264, 2015.

90. Ott PA, Hodi FS and Robert C: CTLA-4 and PD-1/PD-L1 blockade: New immunotherapeutic modalities with durable clinical benefit in melanoma patients. Clin Cancer Res 19: 5300-5309, 2013

91. Kerr KM and Hirsch FR: Programmed death ligand-1 immunohistochemistry: Friend or foe? Arch Pathol Lab Med 140: 326-331, 2016

92. Voutsadakis IA: Immune blockade inhibition in breast cancer. Anticancer Res 36: 5607-5622, 2016.

93. Yi KH and Chen L: Fine tuning the immune response through B7-H3 and B7-H4. Immunol Rev 229: 145-151, 2009. 